\title{
Structural and genetic characterization of fish skeletal muscle
}

\section{tropomyosins}

YOSHIHIRO OCHIAI, AND SHUGO WATABE

Graduate School of Agricultural and Life Sciences, The University of Tokyo, Bunkyo, Tokyo 113-8657, Japan (aochiai@mail.ecc.u-tokyo.ac.jp)

SUMMARY: Tropomyosins were purified from the fast skeletal muscle of several fish species. While their behaviors on two-dimensional PAGE (2D-PAGE) were examined, their thermodynamic properties were compared by differential scanning calorimetry (DSC). Furthermore, full- and partial lengths of cDNAs encoding fast skeletal muscle tropomyosins were cloned from white croaker and walleye pollack, respectively, and their nucleotide and deduced amino acid sequences were determined. It was suggested that the differences observed in the above two analytical parameters among fish species were due to only a few amino acid replacements in the molecule.

KEYWORDS: tropomyosin, fish, fast skeletal muscle, cDNA cloning, primary structure, DSC

\section{INTRODUCTION}

Tropomyosin, found not only in muscle fiber but also in non-muscle cells, is composed of two subunits ( $c$. $33 \mathrm{kDa}$ ) forming two stranded coiledcoil structure. ${ }^{1)}$ In the striated muscle, this is one of the regulatory proteins consisting of thin filaments, lying along the grooves of F-actin. ${ }^{23)}$ Tropomyosin is characterized by its $\alpha$-helical structure throughout the molecule, with a fundamental structural motifs in the arrangement of hydrophobic and hydrophilic residues. ${ }^{4,5)}$ Alternative splicing of one tropomyosin gene generates tissue-specific molecules in higher vertebrates. ${ }^{6,7)}$

Occurrence of isoforms was reported in fish skeletal muscle, ${ }^{8,9)}$ and the primary structures have been deduced for several species. ${ }^{10,11)}$ So far, little information is available on the stability and structure of fish muscle tropomyosins. In the present study, tropomyosins were purified from fast skeletal muscle of several fish species, and their dif- ferences in behaviors on 2D-PAGE and DSC runs were compared. Responsible amino acid residues for the stability difference were also discussed based on the primary structure deduced from cDNA cloning.

\section{MATERIALS AND METHODS}

Tropomyosin was prepared from the acetone-dried powder of dorsal fast skeletal (ordinary) muscle of nine fish species consisting of white croaker Pennahia argentata, walleye pollack Theragra chalcogramma, albacore tuna Thunnus albacares, skipjack Katsuwonus pelamis, horse mackerel Trachurus trachutrus, yellowtail Seriola quinqueradiata, oval filefish Thamnaconus modestus, flounder Limanda yokohamae, and chum salmon Oncorhynchus keta. Tropomyosin was extracted from the acetone powder in a solution containing 1 $\mathrm{M} \mathrm{KCl}$, and purification was carried out mainly by 
the repetition of isoelectric point sedimentation (at $\mathrm{pH}$ 4.5) and ammonium sulfate fractionation (50-60\% saturation). Tropomyosins of high purity $(>95 \%)$ could be obtained without any further purification procedure such as column chromatography.

2D-PAGE was performed using isoelectric focusing in glass capillaries for the first dimension, and SDS-PAGE ( $15 \%$ gel) for the second dimension. Proteins were stained with Coomassie brilliant blue R-250.

DSC was carried out in a medium consisting of $0.1 \mathrm{M} \mathrm{KCl}, 1 \mathrm{mM}$ EDTA, $0.01 \% \mathrm{NaN}_{3}, 1 \mathrm{mM}$ dithiothreitol, $10 \mathrm{mM}$ sodium phosphate buffer $(\mathrm{pH}$ 7) at an approximate protein concentration of 1 $\mathrm{mg} / \mathrm{mL}$ using a MicroCal VP-DSC microcalorimeter.

cDNA cloning was performed as follows: two degenerate oligonucleotide primers were designed based on the highly conserved regions in the amino acid sequences of several vertebrate tropomyosins. ${ }^{10,12)}$ The two primers, 5'-(C/T)TGGAC AA-3' as forward and 5'-GATCT(C/T)TCAGC (A/G)AACTCAGC-3' as reverse (corresponding to $\mathrm{Leu}_{57}-\mathrm{Lys}_{59}$ and $\mathrm{Ala}_{239}-\mathrm{Ser}_{245}$, respectively), were used in PCR for amplification of an expected cDNA fragment of about 700 nucleotides (nt) from the cDNA library. DNA sequencing was performed for both strands with an Applied Biosystems model 373A DNA sequencer after labeling with a Dye Deoxy termination cycle sequencing kit.

\section{RESULTS AND DISCUSSION}

\section{D-PAGE patterns of tropomyosins}

It was revealed that most fish possessed only a single type of subunit ( $\alpha$-type), but those with two isoforms were also recognized in certain species (Fig.1). Scombroid fish (tuna and mackerel), which are already known to have two molecular species ( $\alpha, \beta$ types) of tropomyosin, ${ }^{8,9)}$ were confirmed to do so by 2D-PAGE, though the molecular weights and isoelectric points were clearly different among these fish species. This time, two isoforms of $\alpha$-subunit were also detected in skipjack.

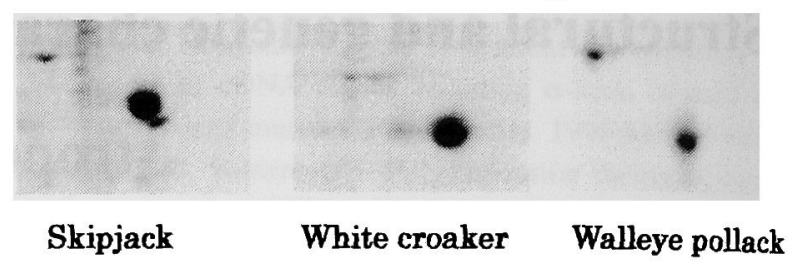

Fig. 1. 2D-PAGE patterns of tropomyosins.

The spots on the left top correspond to actin.

\section{Thermodynamic properties}

An example of DSC pattern is presented in Fig. 2. Two major endothermic peaks were observed at 30.5 and $43.0^{\circ} \mathrm{C}$. Such pattern was also true for all the fish examined except yellowtail and white croaker. The transition temperature for thermal unfolding of fish tropomyosins are summarized in Table 1. For most species, heat-induced conformational changes were completely reversible, as suggested by the observation that DSC patterns were different between the first and second runs. In the case of white croaker, similar curves were obtained for both the first and second runs. These results suggest that white croaker tropomyosin is able to successfully refold to the original structure when measuring temperature is reduced. It could have some relationship to its strong thermal gel-forming ability of muscle. Jackman et al..$^{10)}$ observed different DSC patterns among three types of tropomyosin from salmon muscle.

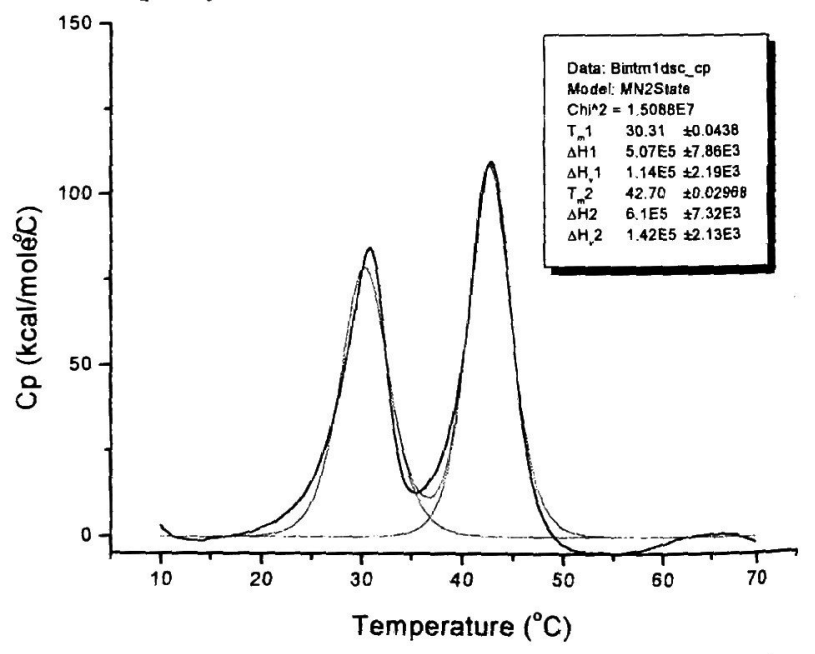

Fig. 2. DSC pattern of albacore tropomyosin. 
Table 1. Transition temperature ( $\mathrm{Tm}$ ) for thermal unfolding of fish tropomyosins

$\left({ }^{\circ} \mathrm{C}\right)$

\begin{tabular}{lccccc}
\hline & \multicolumn{2}{c}{ First measurement } & \multicolumn{2}{c}{ Second measurement } \\
\cline { 2 - 3 } \cline { 6 - 6 } Species & Tm1 & Tm2 & & Tm1 & Tm2 \\
\hline Albacore & 30.5 & 43.0 & & - & 42.9 \\
Skipjack & 31.7 & 41.3 & & 36.1 & 41.8 \\
Horse mackerel & 37.9 & 42.1 & & - & 41.5 \\
Yellowtail & 44.5 & - & & 44.5 & - \\
Chum salmon & 38.7 & 51.3 & & 38.9 & 46.2 \\
White croaker & 44.0 & - & & 44.0 & - \\
Oval filefish & 42.5 & 46.8 & & 42.0 & 46.6 \\
Flounder & 41.7 & 48.5 & & 41.8 & 48.6 \\
Walleye pollack & 32.8 & 44.7 & & 32.8 & 44.7 \\
\hline
\end{tabular}

\section{Structure of the tropomyosin molecule}

The cDNA clone contained an open reading frame of 852 nucleotides encoding 284 amino acids. The deduced amino acid sequence is shown in Fig. 3. The number of amino acid residues coincides with those from most tropomyosins so far reported. $^{5,10,13)}$ White croaker skeletal muscle is considered to have only $\alpha$-type tropomyosin as do many other fish species. ${ }^{8,9)}$ This was also ascertained by 2D-PAGE (data not shown). The molecular weight and isoelectric point were calculated to be 32,728 and 4.55 , respectively. This tropomyosin was rich in ghtamic acid (56 residues per molecule), lysine (39), leucine (34), and alanine (33), contained a single residue each of cysteine and phenylalanine, and lacked tryptophan. In comparison with other $\alpha$-type tropomyosins, the sequence identity was in the range of $94-97 \%$. The sequences of $\mathrm{N}$-terminal 8 residues (MDAIKKKM) were the same irrespective of the sources including invertebrates. $\mathrm{N}$-terminal 9 residues are considered to be important for intermolecular overlapping by the recent NMR studies. $^{13)}$ On the other hand, the predicted secondary structure of white croaker tropomyosin suggested the presence of $\alpha$-helical region along all over the molecule, with four short parts of sheet structure connected with 10 turns. The hydropathy plot was essentially the same with that of rabbit tropomyosin $\alpha$ subunit.

White croaker tropomyosin is considered to possess a coiled-coil structure composed of two $\alpha$ helices with the heptad repeats $(a-b-c-d-e-f-g)_{\mathrm{n}}$, where residues $a$ and $d$ tend to be occupied by hydrophobic residues with 28 -amino acid repetition. The heptad repeats were found to become more highlighted when alanine was assumed to be hydrophobic, though its hydrophobicity is very low. Incidentally, the residues at positions $a$ and $d$ are considered to be responsible for the stability difference of tropomyosin. ${ }^{14)}$ Similar sequential repeats are also present in other proteins with a coiled-coil structure, such as myosin. ${ }^{15,16)}$

Residue bias in the heptad repeats was recognized for hydrophobic amino acids. Namely, out of 33 alanine residues, 13 occupied position $d$, whereas 8 appeared in position $a$. Such bias was also recognized for methionine and leucine. The former appeared only in position $a$ ( 5 out of 7 residues). The latter (34 in total) occupied 16 at position $a$ and 9 at position $d$. On the other hand, a significant heterogeneity was found in the residue

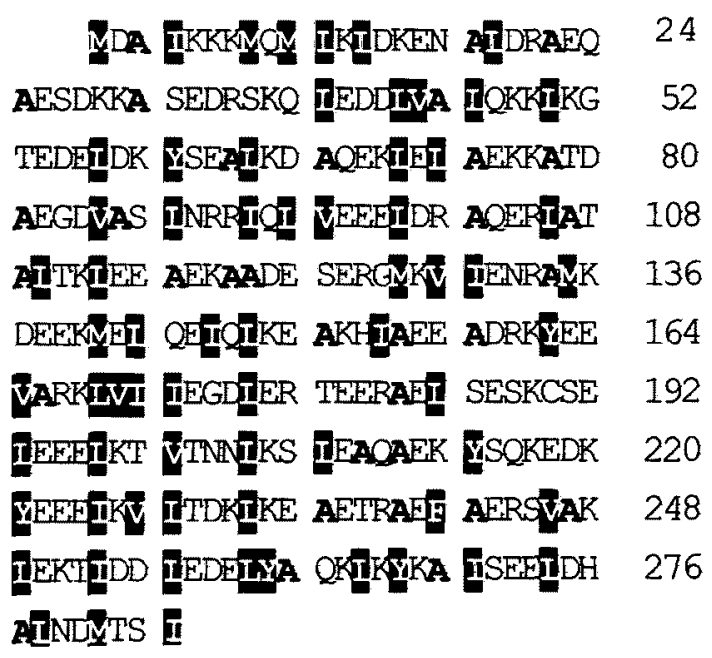

Fig. 3. Deduced amino acid sequences of white croaker tropomyosin arranged in 28-residue repeated segments. ${ }^{11)}$ The residues in white letters against black background are hydrophobic ones. Alanine is boldfaced as a group of hydrophobic residue. 
27 , where amino acids were completely different from each other among different animal species. Namely, this residue is glycine for walleye pollack, serine for white croaker, and alanine for zebrafish (GenBank/EMBL/DDBJ accession no. AI415939). It is likely that these residues are involved in the species-specific difference of tropomyosin.

\section{ACKNOWLEDGMENTS}

The authors are grateful to Dr. M. Nakaya, Dr. D. Funabara, Mr. M. N. Ahsan, Mr. M.C. Huang, Mr. H. Fukushima in our laboratory, and Ms. K. Ahmed in Ibaraki University for technical assistance. This study was supported in part by a Grant-in-Aid from the Ministry of Education, Culture, Sports, Science and Technology of Japan.

\section{REFERENCES}

1. Smillie LB. Structure and functions of tropomyosin from muscle and non"muscle sources. Trends Biochem. Sci. 1979; 4:151-155.

2. Borovikov YS. Conformational changes of contractile proteins and their role in muscle contraction. Int. Rev. Cytol. 1999; 189: 267-301

3. Muthuchamy M, Rethinasamy P, Wieczorek DF. Tropomyosin structure and function. New insights. Trends Cardiovascular Medicine 1997; 7: $124-128$.

4. McLachlan AD, Stewart M, Smillie LB. Sequence repeats in $\alpha$ tropomyosin. J. Mol. Biol. 1975; 98: $281-291$.

5. Iwasaki K, Kikuchi K, Funabara D, Watabe S. cDNA cloning of tropomyosin from the anterior byssus retractor muscle of mussel and its structural integrity from the deduced amino acid sequence. Fisheries Sci. 1997; 63: 731-734.

6. Ruiz-Opazo N, Nadal-Ginard B. $\alpha$-Tropomyosin gene organization. Alternative splicing of duplicated isotype-specific exons accounts for the production of smooth and striated muscle isoforms. J. Biol. Chem. 1987; 262: 4755-4765.

7. Lemonnier $M$, Balvay L, Mouly V, Libri D, Fiszman MY. The chicken gene encoding the $\alpha$ isoform of tropomyosin of fast-twiteh muscle fibers: organization, expression and identification of the major proteins synthesized. Gene 1991; 107: 229-240.

8. Seki N, Iwabuchi S. On the subunit composition of fish. Bull. Japan. Soc. Sci. Fish. 1978; 44: 1333-1340.

9. Heeley DH, Hong C. Isolation and characteriz. ation of tropomyosins from fish muscles. Comp. Biochem. Physiol. 1994; 108B: 95-106.

10. Jackman D, Waddleton DM, Younghusband B, Heeley DH. Further characterization of fast, slow and cardiac muscle tropomyosins from salmonid fish. Eur. J. Biochem. 1996; 242: 363-371.

11. Ochiai $\mathrm{Y}$, Ahmed $\mathrm{K}$, Ahsan MN, Funabara D,Nakaya $M$, Watabe $S$. cDNA cloning and deduced amino acid sequence of tropomyosin from fast skeletal muscle of white croaker Pennahia argentata. Fisheries Sci. 2001; 67: 559-561.

12. Mak AS, Smillie LB, Stewart GB. A comparison of the amino acid sequences of rabbit skeletal muscle $\alpha-$ and $\beta$-tropomyosins. J. Biol. Chem. 1980; 255 : 3647-3655.

13. Greenfield NJ, Hitchcock-DeGregori SE. The stability of tropomyosin, a two-stranded coiledcoil protein, is primarily a function of the hydrophobicity of residues at the helix-helix interface. Biochemistry 1995; 34: 16797-16805.

14. McLachlan AD, Karn J, Periodic charge distributions in the myosin rod amino acid sequence match cross-bridge spacings in muscle. Nature 1982, 299: 226-231.

15. Togashi M, Kakinuma M, Hirayama $Y$, Fukushima H, Watabe S, Ojima T, Nishita K. cDNA cloning of myosin rod and the complete primary structure of myosin heavy chain of walleye pollack fast skeletal musele. Fisheries Sci. 2000; 66: 349-357.

16. Yoon SH, Kakinuma M, Hirayama Y, Yamamoto J, Watabe S. cDNA cloning and characterization of the complete structure for myosin heavy chain of white croaker fast skeletal muscle. Fisheries Sor. $2000 ; 66: 1163-1171$. 